\title{
Privacy-preserving data publishing through anonymization, statistical disclosure control, and de-identification
}

\author{
Grigorios Loukides \\ Department of Informatics, King's College \\ London, UK \\ grigorios.loukides@kcl.ac.uk
}

\author{
Nik Lomax \\ School of Geography, University of Leeds, \\ UK \\ n.m.lomax@leeds.ac.uk
}

\section{Introduction}

Recent developments in information technology allow the collection of massive amounts of data about individuals. These data capture a multitude of activities, characteristics, and aspects of the life of individuals, ranging from demographic, to financial and to health information. For instance, the UK government collects, through various agencies and organisations (e.g., the Office for National Statistics), data related to tourism, crime, investment, business and economy [1]. Data collected by the Office of National Statistics include tabular data (statistical tables), produced from administrative sources or surveys, as well as microdata (data where each record corresponds to a different individual and is associated with a fixed, typically small number of attributes) produced from social surveys.

The use of the collected data is a valuable source for analyses, ranging from answering statistical (aggregate) queries to building statistical models for prediction and classification [2]. This brings significant benefits to researchers and practitioners who analyse the data and to the society at large. At the same time, however, there are considerable concerns regarding violations of personal privacy and misuse of the collected data. For example, $61 \%$ of UK citizens say they have trust and confidence in the NHS or local GP to store and use their personal information while the percentage drops to $53 \%$ and $49 \%$ for the police and national government departments and organisations, respectively [3]. Results of survey in other countries indicate similar concerns. For example, in New Zealand [4], survey participants "expected information provided to government departments to be shared with other government departments", and "gauged acceptability primarily on the need for the information".

To address these privacy concerns, it is important to consider the entire lifecycle of data when protecting them [5]. This includes the collection of data from individuals or businesses (e.g., during surveys), the dissemination of data to a number of authorised parties (e.g., sharing data between governmental organisations), and the public dissemination of data (e.g., as a set of statistical 
tables that are accessible from a website). Fortunately, there are a large number of technological solutions that have been proposed for protecting the privacy of data about individuals. These include formal privacy models, algorithms, protocols, languages, architectures, and software tools [6]. In addition, privacy protection is a legal requirement, which is posed by worldwide legal frameworks, including the Data Protection Act in the United Kingdom and the GDPR in the European Union. Closely related to the enforcement of these frameworks, are recent data governance efforts. These include the establishment of the so-called safe havens, i.e., environments which allow researchers to securely access data. Examples of safe havens are the Population Data BC in Canada and the Farr Institute in the United Kingdom [7].

The efforts to protect the privacy of data have shaped the area of data privacy. An important subarea of data privacy, termed privacy-preserving data publishing [8], concerns the protection of data prior to their dissemination, to a set of authorised data users or (more commonly) to a larger set of unknown data users (e.g., the visitors of a website containing the results of a statistical survey). This chapter will provide an overview of methodological developments in the area of privacy-preserving data publishing, focusing on data anonymization and statistical disclosure control methods:

Data Anonymization: This technique aims to prevent the identification or the inference of sensitive information of a person or organisation from the disseminated data. The disseminated data comes in various forms, including microdata (records of individuals containing, so that each record contains the values of individuals over a small set of features) and various forms of databases. Examples are databases recording, for each individual, a varying and typically very large number of values corresponding to purchased products or visited locations [9], [10], as well as databases recording the social relationships between users in a social network [11]. We will review various methods for performing data anonymization and statistical disclosure control. For example, we will review algorithms for anonymizing microdata [8], [12], set-valued data (where each individual is associated with a large set of values of varying size) [9], [13]-[16], and more complex data comprised of both microdata (relational attributes) and set-valued attributes [17], [18]. For these algorithms, we will also examine implementations in open source software tools [19]-[22].

Statistical Disclosure Control: This technique aims to prevent the identification of a person or organisation from the disseminated data. The disseminated data typically comes in the form of 
one or more statistical tables, resulting from survey or administrative data, or microdata. Statistical disclosure control methodologies are routinely used by national statistical agencies all over the world [23]. We will review commonly used methods for statistical disclosure control, for tabular data and for microdata, focusing on techniques for transforming the data prior to their release. We will also review implementations of these methods in open source software packages [24][26].

Pseudonymization and Hashing: Data anonymization and statistical disclosure control methodologies operate on quasi-identifiers (also known as key variables) and sensitive attributes (also known as sensitive variables). Quasi-identifiers are attributes that in combination can lead to the disclosure of the identity of individuals (a privacy threat termed identity disclosure, or reidentification), such as demographics. Sensitive attributes are attributes that individuals are not willing to disclose (e.g., salary). Data collected from individuals though has typically other attributes that can uniquely identify an individual. These attributes are called direct identifiers and include attributes such as various ids, phone numbers, and emails. Clearly, such attributes need to be protected before applying data anonymization or statistical disclosure control methodologies. One way is to simply remove such attributes from the data. We will briefly examine a different approach, namely pseudonymization with a focus on hashing algorithms.

Last, we will conclude the chapter with a brief overview of the article and some suggestions for future work.

\section{Background on privacy-preserving data publishing}

In the following section, we will present some key terms regarding the data and its protection. The terms are used by multiple techniques and serve as introductory material (foundations) to these techniques.

Types of data: The data can be categorised into structured data, unstructured data, and metadata. Structured data conform to a certain/fixed form, also called schema. For example, as structured data, we can think of a statistical table, or microdata comprised of 100 records (rows) and 5 attributes (columns). Each row in the microdata needs to have values (including empty values) over all 5 attributes. Unstructured data have a loose/flexible schema or no schema. For example, as unstructured data, we can think of a text document, which can contain any number of different words. Metadata are data that describe data (e.g., when different sets of microdata 
are stored in a database, the database keeps information about the number of attributes each of these microdata have). In this chapter, we will deal with structured data, which has been the focus of most of the research in privacy-preserving data publishing, at least from the computer science/statistics community.

Types of attributes based on their values: Within a structured dataset, there are also multiple types of attributes based on their value. The most common ones are numerical (e.g., the age of an individual), categorical (e.g., the ethnicity of an individual), and set-valued attributes (e.g., a set of purchases made by an individual). There are of course more complex types of attributes, where a set can have a certain order or spatiotemporal information (e.g., the purchases made by an individual at each shop and at certain times). In this chapter, we will focus on numerical, categorical, and set-valued attributes.

Types of attributes based on their role in privacy threats: Typically, there are three different types of attributes in structured data based on their role in privacy threats: direct identifiers, quasiidentifiers, and sensitive attributes [8], [23]. Direct identifiers uniquely identify individualsexamples of these attributes are names and phone numbers. Quasi-identifiers, on the other hand, are attributes which in combination identify an individual. Such attributes are demographics (e.g., a 20 year old male living in a house with a certain postcode) or other attributes taking a large set of values (e.g., purchases with a credit card performed by an individual). Last, sensitive attributes are those that individuals are not willing to be associated with. Examples of sensitive attributes are serious diseases, such as psychiatric diseases and forms of cancer, or attributes like income, salary, and receipt of social benefits. Quasi-identifiers are also known as "key variables" and sensitive attributes are also known as "sensitive variables". There may be also attributes that are not classified as direct identifiers, quasi-identifiers, or sensitive attributes, and their inclusion in a dataset poses zero or very low risk to privacy.

Types of attacks to privacy: When dealing with structured data, there are two main attacks; identity disclosure and attribute disclosure [27].

Identity disclosure (or re-identification) occurs when an individual is uniquely associated with their record in published microdata. This is performed even when data are devoid of direct identifiers, since quasi-identifiers are contained in both the published microdata and in publicly available external data sources. For example, it has been estimated that over $87 \%$ of U.S. citizens can be 
re-identified based on a combination of only three demographics (5-digit zip code, gender and date-of-birth) [28]. Many other identity disclosure incidents have also been reported [29]. For instance, in one attack students re-identified individuals in the Chicago homicide database by linking it with the social security death index.

Attribute disclosure (or sensitive information disclosure) occurs when an individual is associated with information about their sensitive attributes. As discussed above, there are common types of attributes that are widely regarded as sensitive. The specification of sensitive attributes is generally left to data owners (e.g., the salary of certain employees is publicly available, and there is no reason for them to try to protect it).

\section{Data anonymization}

We provide a brief discussion on privacy models for anonymization. The focus has been on syntactic models which are implemented by the software that we will survey later. There is another, very important category of privacy models, referred to a semantic models - one of which is differential privacy. We refer to [30]-[32] for works of semantic models.

(Syntactic) privacy models for anonymization: We will discuss privacy models based on the two attacks they aim to guard against; identity disclosure, and attribute disclosure. All these models are applied to record-level data (e.g., microdata).

Models against identity disclosure: The most popular privacy model for protecting microdata from identity disclosure (or re-identification) is k-anonymity [28]. k-anonymity requires each record in a dataset to contain the same values in the set of Quasi-IDentifier attributes (QIDs) with at least k1 other records in the dataset. k-anonymity protects from identity disclosure, because it limits the probability of attackers to successfully link an individual to his or her record, based on QIDs, to $1 / \mathrm{k}$.

There are many variations of k-anonymity. A variation is a privacy model, called k-map [33]. kmap considers that the linking is performed based on larger datasets (called population tables), from which microdata has been derived. Thus, k-map can be satisfied with lower data transformation than what is required by k-anonymity, but provides less protection. Another variation is privacy-constrained k-anonymity. This model assumes that certain combinations of quasi-identifiers are known to an attacker and protects identity disclosure attacks, based on these 
known combinations. A third variation is $\mathrm{k}^{\wedge} \mathrm{m}$-anonymity [13], which is applied to set-valued data (i.e., data in which each individual is associated with a set of values, or belonging to the same attribute, such as "purchase"). $\mathrm{k}^{\wedge} \mathrm{m}$-anonymity aims at preventing re-identification by limiting the probability of re-identifying any individual, based on any set of $m$ or fewer values, to $1 / k$. A last variation is $\left(\mathrm{k}, \mathrm{k}^{\wedge} \mathrm{m}\right)$-anonymity [18], which is applied to records comprised of both microdata (i.e., relational attributes, such as demographics, which have a single value, as well as set-valued attributes, such as the set of purchased products of an individual). ( $\left.k, \mathrm{k}^{\wedge} \mathrm{m}\right)$-anonymity aims at preventing re-identification by limiting the probability of re-identifying any individual, based on all relational attributes and any set of $m$ or fewer values in the set-valued attribute, to $1 / \mathrm{k}$. Note that $\left(\mathrm{k}, \mathrm{k}^{\wedge} \mathrm{m}\right)$-anonymity implies $\mathrm{k}$-anonymity and $\mathrm{k}^{\wedge} \mathrm{m}$-anonymity for the relational and the set-valued attribute, respectively, but the reverse does not hold. That is, applying k-anonymity to the relational attributes and $k^{\wedge} \mathrm{m}$-anonymity to the set-valued attribute independently does not satisfy $\left(k, k^{\wedge} m\right)$-anonymization nor prevents from re-identification as applying $\left(k, k^{\wedge} m\right)$-anonymity does.

Models against attribute disclosure: The most popular privacy model that thwarts attribute disclosure attacks in microdata is I-diversity [27]. It requires each anonymized group in a dataset to contain at least I "well represented" (e.g., distinct or not "too" frequent) sensitive attribute values. The main idea behind I-diversity is to prevent an attacker, who already knows the values of the quasi-identifiers of an individual (or they can identify the anonymized group where the record of the individual belongs) from finding the sensitive value of an individual with sufficiently high probability. Other principles that thwart attribute disclosure, similarly to I-diversity, are $(a, k)$ anonymity [34] and p-sensitive-k-anonymity [35]. They combine k-anonymity with a weak notion of I-diversity, where each anonymized group must have at least I distinct values in the sensitive attribute. t-closeness [36] is another privacy model for protecting demographics from attribute disclosure. This model prevents an attacker, who has the same knowledge about quasi-identifiers as I-diversity, from learning information about an individual's sensitive value that is not available from the entire published dataset. In addition, I-diversity has been adapted to set-valued data. For example, in [37], the privacy model of PS-rules is proposed. Each PS-rule is an implication of the form $X \rightarrow Y$, where $X$ and $Y$ are combinations of values. A PS-rule is protected when the probability of $X$ is sufficiently large and the probability that $Y$ can be inferred from the data, given $X$, is sufficiently small. The latter probability prevents attribute (sensitive information) disclosure, in the same way I-diversity does for relational attributes. 
Data transformation techniques for anonymization: The transformation of data is applied to quasi-identifiers, in order to prevent identity disclosure. To transform data, (a) microaggregation [38], (b) generalization [28], and (c) suppression [34] are typically applied. All these techniques have been implemented in popular software tools. Microaggregation involves replacing a group of quasi-identifier values using a summary statistic (e.g., median) and is applicable to demographics but not to diagnosis codes. Generalization, on the other hand, suggests replacing quasi-identifier values by more general, but semantically consistent, values. There are different types of microaggregation, generalization and suppression, which typically work better for certain types of attributes (e.g., numerical vs. categorical), as well as additional data transformation techniques such as anatomy (bucketization) [21]; please see [8] for a survey.

Discussion of anonymization algorithms: Algorithms for anonymization work by (i) employing a privacy model, and (ii) applying one or more data transformation techniques, to achieve privacy, while also preserving the utility of the data. Unfortunately, the problem of achieving a desired level of privacy according to the specified privacy model and at the same time maximizing utility is computationally infeasible. Therefore, most algorithms employ heuristics for solving the problem and produce sub-optimal solutions.

The heuristics intuitively depend on the form of data that they transform. So, we can classify the algorithms, based on whether their heuristics are applied to relational attributes (microdata) or they are applied to a set-valued attribute. This gives two categories of algorithms. A third category of algorithms uses both types of heuristics, to anonymize complex data comprised of relational and set-valued data. In the following, we review each category of algorithms; anonymization algorithms for relational data, for transaction data, and for both relational and set-valued data.

\section{Algorithms for relational data (microdata)}

In the following, we will provide a classification of algorithms for relational data based on the type of heuristics (i.e., search strategies) they employ to transform microdata into an anonymized dataset. The classification was first provided in [39]. We will classify these algorithms into those: that employ:

(i) binary search on the lattice of possible ways to transform the data by generalizations,

(ii) lattice search similar in principle to the Apriori association rule mining technique,

(iii) genetic search on the lattice of possible generalizations,

(iv) data partitioning, and 


\section{(v) data clustering.}

The main idea behind strategies (i)-(iii) is to represent the possible ways to generalize a value in a quasi-identifier attribute, using a taxonomy, and then combine the taxonomies for all quasiidentifier attributes, to obtain a lattice. For instance, Greek and Italian are the leaf-level nodes of a taxonomy for Nationality and their immediate ascendant is the generalized value European. Similarly, Black, White, and Asian are the leaf-level nodes of a taxonomy for Race, whose root value and immediate ascendant of the leaves is the most generalized value "Any". Thus, we can combine these two taxonomies to get a lattice for Nationality and Race. Each node in this lattice represents a different set of generalized values for Nationality and Race, such as $\{$ Greek, White\}, \{Greek, Black\}, \{Greek, Asian\}, \{Italian, White\}, etc. Thus, finding a way to generalize values can be performed by exploring the lattice using heuristics that avoid considering certain lattice nodes for efficiency reasons. The strategy (i) prunes the ascendants of lattice nodes that are sufficient to satisfy a privacy model, while the strategies (ii) and (iii) prune lattice nodes that are likely to incur high utility loss. The latter nodes are identified while considering nodes that represent incrementally larger sets of generalized values, for strategy (i), or while selecting nodes by combining their descendants, as specified by a genetic algorithm, in the case of strategy (ii).

Two popular algorithms that employ lattice search and have been implemented into various software tools for anonymization are Incognito and Incognito and Full subtree bottom-up generalization. Their operation is briefly summarized below

- Incognito [12]: It gets as input microdata (i.e., a set of records, each of which corresponds to a different individual, a set of quasi-identifier attributes, a set of taxonomies for the attributes, and a parameter k for employing k-anonymity). It employees k-anonymity, with the specifying value, using lattice-based search on the lattice constructed by combining all the taxonomies for the quasi-identifier attributes. For efficiency, it uses several heuristics that avoid searching the entire lattice. Incognito has led to the development of variants based on lattice-search, which were more suited to anonymize biomedical microdata, such as Optimal Lattice Anonymization (OLA) [68]. There are three issues/limitations with Incognito (and its variations). The first limitation is that it cannot be applied to numerical attributes with large domains, because it only applies to attributes with taxonomies, and attempts to discretize such attributes result in utility loss (e.g., a number 11.25 would be replaced with a range of values [11-12] in the taxonomy). The second limitation is that it does not explore a large number of generalizations, because if 
the hierarchies are large, the lattice becomes much larger than the hierarchies (exponential blow-up) and then the algorithm becomes slow. The third issue is that the algorithm uses a rather crude objective measure to capture the amount of utility lost during anonymization. Thus, its result in terms of utility (i.e., solution) is not very good.

- Full subtree bottom-up method [40]: This method traverses the hierarchy tree from bottom to top specializing every attribute in its parent value. Specialization is the opposite operation of generalization (i.,e, the values are replaced by more specific values - e.g, the value Asian is replaced by the value Chinese in Ethnicity). This algorithm operates on the set of hierarchies of the quasi-identifier attributes, instead of the lattice, and it uses a utility criterion that is more fine-grained than that of Incognito. Thus, it outperforms Incognito in terms of both privacy and utility. In addition, it avoids the expensive lattice search of Incognito, being substantially faster.

- Top-down specialization [41]: This method initially generalizes every value of every quasiidentifier attribute to the root node of the taxonomy (i.e., to the most general/abstract value "Any"), and then it traverses the taxonomy from top to bottom specializing each value to its children in the taxonomy. Thus, it can be seen as the "opposite" of the Full subtree bottom-up method. The specialization is performed at one quasi-identifier attribute at a time. Thus, an ordering for selecting the quasi-identifier attribute whose values will be specialized is needed. The top-down specialization algorithm can use various heuristics for this. For example, there are heuristics which help producing data that are good for answering aggregate queries, and others which help producing data that are good for preserving the accuracy of data mining tasks such as classification [42]. The algorithms is also relatively fast (faster that Incognito). Furthermore, it is one of the few algorithms that have been implemented for Big Data, which do not fit into the main memory of a computer [43], [44].

However, algorithms based on strategies (i) and (ii), such as Incognito and Full subtree bottomup method explore a small space of potential solutions and thus may fail to preserve data to the extent that genetic search strategies can do. In general, genetic search algorithms work by starting from a set of random (or seed) solutions and then iteratively combine these solutions, or tweak them slightly, in order to produce better solutions. Their limitation is that they are computationally intensive and may converge slowly to a good solution (i.e., they need a large 
number of iterations). Consequently, more recent research has focused on developing methods that use strategies (iv) and (v), which are applied to the records of a dataset, and not to attribute values as strategies (i)-(iii) are. The objective of the former strategies is to organize records into carefully selected groups that help the preservation of privacy and the satisfaction of a utility objective. Both data partitioning and clustering-based strategies create groups iteratively, but they differ in the task they perform in an iteration. Specifically, partition-based strategies split records into groups, based on the value that these records have in a single quasi-identifier attribute (i.e., an iteration creates two typically large groups of records that are similar with respect to a quasiidentifier), while clustering-based strategies merge two groups of records, based on the values of the records in all quasi-identifier attributes together. Therefore, partitioning-based methods tend to incur higher utility loss when compared to clustering-based methods, and they are sensitive to the choice of the splitting attribute, performing poorly particularly when the dataset is skewed. However, partitioning is faster than clustering by orders of magnitude [45]. In the following, we briefly present two popular algorithms that have been implemented into software tools and use partitioning and clustering, respectively. The partitioning based method is called Mondrian and the clustering based method is called Cluster:

- Mondrian [45]: This method performs a multidimensional partitioning of the space of quasi-identifier attributes. Simply put, it selects the quasi-identifier attribute that has the largest domain size, and splits the records into two parts. The first part has values smaller than the median value of the selected attribute, and the second part has the remaining values. Then, the splitting is performed recursively, to each of the two created groups, as long as each group contains at least $k$ and at most $2 k-1$ records. For example, in the first step of recursion, the group that has values smaller than the median of the first selected attribute is partitioned into two groups, based on the median value of the second attribute. After the partitioning ends, the values in each group are anonymized independently, using generalization. There are many ways to do this. For example, in a numerical attribute, we can replace all the values in the attribute of the group with their range. Or, we can replace them with a summary statistic such as mean or median. If the attribute is categorical, we can replace the values in the attribute of the group with a set containing all values, or, if we have a taxonomy for the attribute, we can replace the values in the group with their closest common ancestor in the taxonomy. Despite its simplicity, Mondrian is a very effective and efficient algorithm. It is effective, because it adopts a flexible way to generalize data which avoids the need for taxonomies. It is efficient, because it only 
considers one attribute at a time and finding the median and splitting the groups into records is fast.

- Cluster [18], [46]: This method works in two phases. First, it performs clustering (i.e., grouping) of the records based on their values in the quasi-identifier attributes. The main idea is that a cluster should contain records that are similar with respect to their quasiidentifier values (e.g., a group contains the values 51 to 55 in a quasi-identifier attribute Age). This is because, in the second phase, the algorithm performs generalization to the values in the quasi-identifier attributes of each group separately. Since generalization in a numerical attribute replaces all values in a group with their range, having a group with similar values will result in a small range that, intuitively, preserves data utility well. Similarly, in a categorical attribute, generalization replaces the values in a group by their set of their closest common ancestor in the taxonomy. Thus, having a group with similar values with respect to a categorical attribute results in an anonymized group that has a small set (few values) in the attribute, or a closest common ancestor that is not too general (i.e., it is relatively low in the taxonomy). To do the clustering, the algorithm selects a random record as a seed and finds $\mathrm{k}-1$ other records with values in the quasi-identifier attributes (all quasi-identifier attributes are considered together) to that seed. Then, it forms a cluster of k records, comprised of the seed and the remaining k-1 records. After that, the process is repeated, and a new cluster is created. The process continues until every record is clustered. The last property may require adding the left over records, by the cluster creation phase, in their closest cluster, with respect to a similarity measure. After the clusters are formed, anonymization is performed to the quasi-identifier attributes of each cluster independently. This gives much flexibility in the way anonymization is performed. Similar to Mondrian, there is no need for hierarchy and each group (subpartition in the case of Mondrian, and cluster in the case of the Cluster algorithm) is anonymized separately. However, Cluster tends to perform better in terms of preserving data utility, because the cluster creation phase takes into account all attributes at the same time, rather than a single attribute at a time as it is being done in the case of Mondrian. The downside is the large runtime of the Cluster algorithm, and the much worse scalability in terms of the cardinality (number of records) in the dataset.

The aforementioned algorithms employ k-anonymity but they can easily be modified to additionally enforce I-diversity or t-closeness. The modification involves an extra check during the 
search strategy (lattice search, partitioning or clustering), to ensure that the resultant groups or clusters, satisfy l-diversity or t-closeness.

\section{Algorithms for anonymization of set-valued data}

In the following, we will provide a classification of algorithms for set-valued data based on the type of heuristics (i.e., search strategies) they employ to transform the data in order to anonymize them. We will classify these algorithms into those: that employ:

(i) space partitioning in a bottom-up or top down fashion,

(ii) data partitioning in a top-down, vertical or horizontal fashion, and

(iii) space clustering.

Notice that these algorithms also use clustering and partitioning but, contrary to the algorithms for microdata, the clustering and partitioning may be performed on either the space of values of the set-valued attribute, or to the data. Notice also that some search strategies used in algorithm for relational data do not apply to algorithms for transaction data. For example, lattice search cannot be used in the context of a set-valued attribute, because there is a single, set-valued attribute to consider and thus we cannot construct a lattice. However, we can still use a single taxonomy which organizes all values of the set-valued attribute (i.e., all different objects that can be purchased by an individual, in a set-valued attribute "purchases"), to model the ways these codes can be generalized.

The main idea behind algorithms that employ space partitioning techniques is to partition the set of all possible values in the set-valued attribute (e.g., set of all possible products that can be purchased) into smaller subsets, and then to anonymize each record by replacing each value with one of the subsets. For example, if a record contains the products $\{a, c\}$ from a set of all possible products $\{a, b, c, d, e\}$, which is partitioned into $\{a, b\}$ and $\{c, d, e\}$, the algorithms will replace "a" with $\{a, b\}$ and "c" with $\{c, d, e\}$, producing an anonymized record $\{a, b\}\{c, d, e\}$. This essentially injects uncertainty, because the data recipients would not be certain whether $\{a, b\}$ represented $a$ or $b$, or $a$ and $b$ together, in the data before anonymization. Clearly, space partitioning can be performed in two ways; bottom-up, where the algorithm tries to create subsets by merging/combining smaller subsets (e.g., it tries to combine "a" and "b", to create the subset $\{a, b\})$, or top-down, where the algorithm tries to create subsets by splitting larger subsets (e.g., it tries to split $\{a, b, c, d\}$ into $\{a, b\}$ and $\{b, c, d\})$. A popular algorithm that employs space partitioning techniques for anonymizing set-valued data and has been implemented into software for 
anonymization is Apriori Anonymization. A brief overview of the operation of Apriori Anonymization is presented below:

- Apriori Anonymization [13]: This method enforces $k^{\wedge} m$-anonymity. To achieve this, it employs bottom-up space partitioning. The partitioning technique begins with subsets comprised of one item. If $\mathrm{k}^{\wedge} \mathrm{m}$-anonymity is not satisfied, for a given $\mathrm{k}$ that is an input parameter, the subsets are merged, to create larger subsets, and then the algorithm checks whether or not $\mathrm{k}^{\wedge} \mathrm{m}$-anonymity is satisfied. This is performed iteratively, until $\mathrm{k}^{\wedge} \mathrm{m}$ anonymity is satisfied. The larger subsets are specified using a taxonomy that is given as input. So, in the first iteration, "a" may be merged with "b", to create $\{a, b\}$, and in the second iteration $\{a, b\}$ may be merged with $\{c, d\}$, to create $\{a, b, c, d\}$. The main idea is that further merging increases the frequency of a subset in the dataset, so it will eventually guarantee $k^{\wedge} \mathrm{m}$-anonymity.

The Apriori Anonymization algorithm was one of the first attempts to anonymize set-valued data, and it led to subsequent research in the field. For example, it led to data partitioning algorithms which require a smaller amount of memory and they are substantially faster than Apriori Anonymization. As mentioned above, data partitioning algorithms work in a top-down fashion and can further be categorized into horizontal or vertical partitioning algorithms. In the following, we provide a brief overview of two popular anonymization algorithms for set-valued data that employ data partitioning, namely Vertical Partitioning Anonymization (VPA), and Local Recoding Anonymization (LRA):

- VPA (Vertical Partitioning Anonymization) [14]: The algorithm first partitions the domain of values (e.g., $\{a, b, c, d\}$ in our example) into subsets (e.g., $\{a, b\}$ and $\{c, d\})$ and then generalizes the values in each subset, using global generalization. Global generalization means that each occurrence of a value in any record of the dataset will be replaced by its corresponding generalized value. For example, any record that has "a" or "b" in the original dataset will have $\{a, b\}$ in the anonymized dataset. Next, VPA merges the generalized items to ensure that the entire dataset satisfies $\mathrm{k}^{\wedge} \mathrm{m}$-anonymity.

- LRA (Local Recoding Anonymization) [14]: The algorithm partitions a dataset horizontally into sets of records that can be anonymized with minimal utility loss, and then generalizes the values in each set separately. 
Although simple and intuitive, the LRA method does not perform well in practice because each set of records contains multiple values and it is difficult to find a set of records with similar values in such a high dimensional space. However, it is easy to parallelize and thus can be used as a simple heuristic for anonymizing large amounts of data (that do not fit into the main memory of a single computer). VPA performs better than LRA and Apriori Anonymization in terms of efficiency and it is also easy to parallelize, as explained in [14]. Furthermore, the anonymized data generated by VPA are similar in terms of utility with those generated by Apriori Anonymization. Thus, among the Apriori Anonymization, LRA, and VPA, the preferred choice should be VPA.

Yet, the space partitioning and data partitioning strategies (Apriori Anonymization, LRA, and VPA) have three limitations that led to the development of more effective algorithms based on space clustering. First, due to the use of $k^{\wedge} m$-anonymity, Apriori Anonymization, LRA and VPA protect all combinations of $m$ values, although some of these values may not be sensitive. For example, if the possible values are $\{a, b, c, d\}$ and $m=2$, every subset comprised of 2 values will be protected based on $k^{\wedge} m$-anonymity. However, "d" may correspond to an item that individuals consider as non-sensitive. The extra protection offered to " $d$ " harms the utility of the data, because it requires extra anonymization. Second, the anonymization of data performed by Apriori Anonymization, LRA, and VPA may not comply with certain requirements that data recipients have regarding the analysis of data. For example, if a required analysis is to compute the frequency of "a" in the dataset, and the anonymized records contain $\{a, b\}$, then we cannot exactly compute the frequency of "a" from the anonymized data, because we are uncertain whether an $\{a, b\}$ in the anonymized data corresponds to "a". A third limitation has to do with the fact that Apriori Anonymization, LRA, and VPA employ a fixed (input) taxonomy for performing the anonymization. So, it may be better to create a subset comprised of "b" and "e", from a data utility perspective, but the algorithm cannot do this if the taxonomy does not include $\{b, e\}$ as a node (i.e., possible way to generalize data). Unfortunately, it is not easy to create taxonomies that lead to high levels of utility, because the construction of subsets depends on the data at hand.

These three limitations of Apriori Anonymization, LRA and VPA led to space clustering techniques. The techniques do not employ $k^{\wedge} \mathrm{m}$-anonymity but a more flexible scheme, in which the subsets that need to be protected are specified as input. For example, if "d" does not require protection, but every other subset of 2 elements in $\{a, b, c, d\}$ does, the input to these algorithms would be $\{a, b\},\{a, c\}$, and $\{b, c\}$. The specified subsets that require protection are called "privacy constraints". Second, these techniques also get as input a list of subsets whose frequency needs 
to be preserved after anonymization (e.g., to allow certain analyses). These subsets are called "utility constraints". If utility constraints are specified, then the anonymized data will be as useful as the original data for these analyses. Third, the space clustering techniques do not use a fixed taxonomy, but instead allow any possible subset of values to be created, as long as this does not violate the protection and utility requirements. By lifting these three limitations, the space clustering techniques are able to produce much more useful data. The downside is the larger exploration space (e.g., every subset must be considered in the worst case, instead of a smaller number of subsets that are specified by the taxonomy), which means worse runtime. In the following, we present a brief overview of two popular space clustering algorithms that have been implemented into software for anonymization, COAT (COnstrained-based Anonymization of Transactions) [16] and PCTA (Privacy-constrained Clustering-based Transaction data Anonymization) [47]:

- COAT (COnstrained-based Anonymization of Transactions) [16]: The algorithm iteratively selects privacy constraints and transforms data to satisfy them. For each privacy constraint, COAT generalizes items in accordance with the specified utility constraints and attempts to minimize information loss, in order to preserve data utility. When a privacy constraint cannot be satisfied through generalization, COAT suppresses (i.e., removes) the least number of items required to meet this constraint.

- $\quad$ PCTA (Privacy-constrained Clustering-based Transaction data Anonymization) [47]: This method performs space clustering, and it gets as input a set of privacy and a set of utility constraints as COAT does. It differs from COAT in the way it processes privacy constraints, and the difference is important for improving both the utility and the runtime of the algorithm. Specifically, it sorts the privacy constraints with respect to their frequency in the data, in decreasing order, and then process each privacy constraint, applying generalization to the values in each privacy constraint. The frequency is measured on the original data, or in the intermediate anonymized data that is being constructed by the algorithm as it runs. The order of processing privacy constraints has been shown experimentally to require less information loss, intuitively, because privacy constraints with large frequency are "close" to having a support of k, which makes them protected. In other words, such privacy constraints can be anonymized without a large loss of data utility. Furthermore, to improve efficiency, the algorithm employs a "lazy updating" strategy, essentially anonymizing a privacy constraint only when it is required. According to the 
experiments that are reported in [47], PCTA was better than Apriori Anonymization in terms of utility by 26 times, and better than COAT in terms of utility by 6 times. The utility was measured by the Average Relative Error of answering statistical queries that count the number of times a certain combination of values appears in the dataset, using publicly available web and marketing data.

Algorithms for anonymization of data containing both relational and set-valued attributes. The problem of anonymizing a dataset containing both relational attributes (e.g., demographics) and set-valued attributes (e.g., a set of purchased products, or yes/no answers in a set of features) is very challenging. The main challenge comes from the fact that these two types of attributes have different characteristics (e.g., a small domain vs a large domain, for a relational and a set-valued respectively, and one vs. multiple values, for a relational and a set-valued, respectively) which require different algorithms for their anonymization. Yet, the problem can be solved by finding a good way to combine the way that the anonymization algorithms for relational and for set-valued data operate. The main idea [18] is to create large clusters (groups of records) with respect to the relational attributes, using one of the algorithms for anonymized data, such as Incognito, or Cluster, and then applying an algorithm for set-valued data to each group of records independently. This naïve strategy, however, has the problem that it is difficult to decide the size of the clusters. Furthermore, a single size may not be adequate, as the goodness of clusters with respect to utility depends on the frequency of values in the dataset. So, more advanced frameworks for anonymizing a dataset with relational and set-valued attributes have been proposed. These frameworks can be instantiated with different algorithms for relational and for set-valued attributes that we surveyed above, and their operation is briefly explained below:

- Rmerger [18]: This framework starts by creating k-anonymous clusters, using an anonymization algorithm for relational attributes. Each cluster should have utility loss less than a threshold that is an input parameter. Then, the algorithm merges together clusters based on their respective relational attributes. The clusters that are merged need to be close together with respect to the values in the quasi-identifier relational attributes. Specifically, the merging works iteratively, merging the pair of the two closest clusters. This is performed until any further merging would violate the threshold. The result is a set of large clusters. Then, the relational attributes in each cluster are anonymized independently, and finally, an algorithm for set-valued data anonymization (e.g.,Apriori Anonymization) is applied to each cluster. The final clusters satisfy $\left(\mathrm{k}, \mathrm{k}^{\wedge} \mathrm{m}\right)$-anonymity. 
- Tmerger [18]: This framework differs from Rmerger in that it merges together clusters based on their respective values in the set-valued attribute. In more detail, it evaluates how many common, different and total values exist between every record of the clusters, and chooses the clusters that have semantically closest items. After the formation of clusters, the anonymization of each cluster is performed in the same way as in the Rmerger framework.

- RTmerger [18]: This framework differs from Rmerger and Tmeger in that, during cluster mergings, it takes under consideration the relational and the set-valued data inside each clusters.

Each of the three frameworks offers a different benefit. Rmerger works best to create anonymized data that allow accurate analyses on the set-valued attribute, but results in more information loss with respect to the relational attribute. Nevertheless, the information loss is bounded by the input threshold. Tmerger on the other hand produces data that allow accurate analyses on the relational attributes but with more information loss in the transaction attribute. RTmerger takes a middle line. Thus, which framework is best depends on the type of analyses that are planned for the dataset to be anonymized. In terms of efficiency, Rmerger was the fastest, and RTmerger the least efficient.

Implementation of anonymization algorithms in software tools: In this section, we review software for anonymizing relational and/or set-valued data. Our focus is on software tools that are open-source or closed-source tools, for which there are published details. This is because, for such software tools, there is accessible knowledge about the algorithms implemented in them. We also focus on the algorithms implemented in each software tool, mentioning the "software engineering" features of each tool, such as input/output and Graphical User Interface (GUI) very briefly. Furthermore, for ease of reference and coherence with the previous sections that reviewed algorithms, we group software based on the algorithms they support.

We start with algorithms for anonymizing microdata (i.e., a table where each record contains a fixed set of (relational) attributes, numerical or categorical). For such data, there are the following software tools: 


\begin{tabular}{|l|l|l|l|l|l|}
\hline Algorithm I Software & $\begin{array}{l}\text { SECRETA } \\
{[19]}\end{array}$ & $\begin{array}{l}\text { UTDA } \\
{[20]}\end{array}$ & $\begin{array}{l}\text { TIAMAT } \\
{[48]}\end{array}$ & $\begin{array}{l}\text { CAT } \\
{[21]}\end{array}$ & $\begin{array}{l}\text { ARX } \\
{[69]}\end{array}$ \\
\hline Incognito & $\mathrm{V}$ & $\mathrm{V}$ & $\mathrm{V}$ & & \\
\hline $\begin{array}{l}\text { Top-down } \\
\text { Specialization }\end{array}$ & $\mathrm{V}$ & & & & \\
\hline Mondrian & $\mathrm{V}$ & $\mathrm{V}$ & & $\mathrm{V}$ & \\
\hline Cluster & $\mathrm{V}$ & & & $\mathrm{V}$ & \\
\hline OLA & & & & & $\mathrm{V}$ \\
\hline
\end{tabular}

Table 1. Anonymization algorithms for microdata and different implementations for them in open source software tools.

A comparison of the tools in terms of the methods they implement

As can be seen from Table 1 above, SECRETA supports all algorithms except OLA, which is supported only by ARX. As mentioned above OLA is a variant of the Incognito algorithm, which is designed for the protection of biomedical data. Incognito and Mondrian turn out to be implemented in most systems, with Top-down specialization being implemented only in SECRETA, and Cluster being implemented in SECRETA and CAT. Since each algorithm has its own benefits in terms of effectiveness and efficiency (e.g., Mondrian is very efficient but Cluster is able to preserve data utility better), and since both effectiveness and efficiency depend on the dataset at hand (i.e., all algorithms are either optimal in terms of utility but with respect to very limited transformation optimality functions, or are heuristics with no approximation guarantees), it is important for a software tool to allow the user to try different algorithms, in an attempt to find the algorithm that performs better for the anonymization task at hand. In this respect, SECRETA is the most suitable software. For example, it offers an "experimenter" interface that allows the user to configure the algorithms and see how all algorithms perform with respect, not only to a utility measure, but also with respect to statistical queries that the user needs the anonymized data to support well. On the other hand, SECRETA focuses on k-anonymization and does not implement versions of Incognito or Mondrian that support I-diversity or t-closeness, to offer protection against attribute disclosure as well. This is a benefit of UTDA (University of Texas at Dallas Anonymization toolkit) and of TIAMAT. These tools implement versions of Incognito that support either I-diversity or t-closeness. A benefit of ARX is that it uses a large variety of functions for measuring risk against re-identification (in addition to k-anonymity) and that it can support various methods of transforming the data (such as generalization, suppression, and 
microaggregation). Its main limitation is that it supports a single algorithm that is able to explore a small part of the solution space, compared to algorithms like Mondrian or Cluster, which generally offer better data utility than lattice-search based algorithms, such as the algorithm implemented in ARX.

\section{A comparison of the tools in terms of software engineering aspects}

One main difference between UTDA and the other tools is that UTDA does not offer a graphical user interface. It is simply a JAVA library, for programmers to use in order to avoid implementing anonymization algorithms from scratch. This means that, to use UTDA, one needs to have programming experience in JAVA. On the contrary, all other tools offer graphical user interfaces. The graphical interface of TIAMAT is quite simple, offering two rather crude quality measures to evaluate the utility of data that have been anonymized by Mondrian or Cluster. The graphical user interface of CAT is again quite limited in terms of evaluating data utility. SECRETA implements a large variety of measures for evaluating data utility, including a tool that allows a user to measure utility based on a workload of statistical queries, as mentioned above. The user interface of SECRETA offers also plots and several visualization tools that can be used to compare the performance of algorithms. The ARX has also a sophisticated graphical user interface, which additionally offers support for measuring the risk of re-identification, using various risk assessment models and privacy principles.

Another "software engineering" aspect of the tools is how well they can be extended to support different algorithms. SECRETA is built in an algorithm-agnostic way, essentially being able to support any algorithm that provides the necessary input to the tool. ARX is also easy to extend, and its team has recently provided support for additional algorithms, such as some methods that enforce the semantic model of differential privacy. The other tools seem very limited in terms of supporting new algorithms; or at least without requiring heavy re-engineering.

A last "software engineering" aspect of the tools is how well they support large data that does not fit into the main memory of a computer. For data in the order of GBs, no tool will be able to perform well. This is an inherent limitation of the algorithms that the tools support, which all are sequential (i.e., they are executed in a single computer, instead of utilizing multiple computers at the same time). Recently, there has been work on parallel anonymization algorithms [] and it would be good to see software tools utilizing such algorithms. 
We now proceed to discussing implementations of algorithms for set-valued and for data containing both relational and set-valued attributes. Currently, the only software tool that has implemented algorithms for set-valued data is SECRETA. Specifically, SECRETA implements the following algorithms:

- Apriori Anonymization

- LRA

- VPA

- COAT

- PCTA

In addition, the only software that has implemented algorithms for relational and set-valued attributes is SECRETA. Specifically, SECRETA offers implementations of the Rmerge, Tmerge, and RTmerge framework [18]. In each framework, any of the implemented algorithms for relational and for set-valued attributes may be used, and the results can be evaluated using a common visualization tool that allows easy benchmarking.

\section{Statistical Disclosure Control (SDC) techniques}

We provide a brief discussion on statistical disclosure control techniques. Most of these techniques have been primarily developed by the statistics community and their objective is to protect the privacy of microdata against re-identification [23]. The focus has been on popular techniques which are used to protect the data and they have implemented by the software that we will survey later. The main idea behind all the operations is to change the values of the attributes, in a controlled way, in order to protect them.

In the following, we briefly present each operation:

- Global recoding: The main idea of global recoding is to reduce the number of possible outcomes of a variable (i.e., make it more general). When applied to a numerical attribute, global recoding replaces its values with intervals (i.e., discretizes the attribute). For example, a value in a quasi-identifier attribute Age that is between 14 and 18 may be recoded as [14-18], implying that an individual's age can take any value between 14 and 18 (inclusive). When applied to a categorical attribute, global recoding replaces its values with more general values (e.g., Greek is recoded to Asian). There are also more 
specialized operations that can be classified as recoding, such as the truncation of the values of a numerical attribute, by removing a specified number of digits from the end. For example, the value 55301 in an attribute Salary becomes $55^{* * *}$, where * can be any digit. This operation is equivalent to replacing 55301 with an interval 55000 to 55999.

- Local suppression [49]: This operation removes combinations of values that have lower frequency than a threshold from the protected (i.e., transformed) data. It is typically applied to categorical quasi-identifier attributes, and its main idea is to reduce risk by removing values that appear infrequently. For example, when a combination of values \{Greek, Asian\} in Nationality and Race, respectively is infrequent in a dataset, but the values $\{$ Greek, White\} are common, local suppression may be applied to remove Asian from the dataset, ensuring that remaining combinations are all sufficiently frequent (e.g., to satisfy k-anonymity with a given k). Individual values are suppressed in a way that the set of variables with a specific pattern are increased.

- PRAM [50]: The Post-RAndomization Method or PRAM is a probabilistic method for disclosure protection of categorical variables in microdata. In the protected (i.e., transformed) microdata, the scores on some categorical variables for certain records are changed to a different score according to a prescribed probability mechanism, namely a Markov matrix. There is also possibility to limit the change to the $n$ nearest values, where $\mathrm{n}$ is a specified parameter. For instance, consider a quasi-identifier attribute Race with the values $\{$ White, Black, Asian\}. The transition of a value from category White to category Black is, for example, fixed with probability 0.7 . This means that only with probability $\mathrm{p} 1=0.13$ can a value of Black be changed to either White or Asian.

- Microaggregation [38], [51]: The main idea behind this operation is to replace a set of attribute values with an aggregate statistic. As in the case of anonymization, the values should be close enough, for the aggregate statistics to be informative (i.e., a good estimate of the values it replaces). Micro-aggregation tries to form groups with similar values in all attributes of a dataset, by taking into account the distances between records. There are several algorithms that result in microaggregated data with a very high level of utility [38]. In addition, there is choice for the aggregate statistic that can replace a set of values. For instance, the median can be used to replace categorical values and the mean can be used to replace numerical values. 
- Top/bottom coding: This operation replaces the top (maximum) and/or the bottom (minimum) values in a numerical attribute with user-specified values. This means that an aggregate category for the top values is created that contains all values deemed large enough, and similarly an aggregate category for the bottom values is created that contains all values deemed small enough. Clearly, this operation can be seen as a special case of "global recoding", where the "recoding" is performed on the top and bottom values only. The intuition behind this operation is that extreme values are rare (e.g., a value over 100 in Age) and thus may allow easier re-identification of individuals.

- Rounding: This operation requires the values of a numerical attribute to be rounded with a user-specified base. For example, the value 36.5 in Age may be rounded to 36 . The intuition behind this operation is that specific digits may pose larger risk of reidentification because they are rarer than the rounded value.

- Noise addition: This operation transforms a value in a numerical attribute by adding a random number (noise) into it. The main intuition is to replace the actual value with a fake one, instead of a more general/abstract value as in generalization. Noise addition can readily reduce the risk of re-identification, and protect from attacks involving simple linkage with external datasets. There are different types of noise that can be added, such as uncorrelated noise (usually normally distributed, with variance proportional to the variance of the attribute whose values are perturbed), or correlated noise. When the noise is uncorrelated, the mean of the attribute is preserved. When the noise is correlated, the mean as well as the variance and correlation coefficients between attributes can be preserved. There are multiple algorithms for adding noise, the majority of which are applied to numerical attribute. The benefits of these algorithms is that they make no assumption about on the range of possible values (e.g., they can be applied to continuous values) and that it may be possible to use the data to answer queries involving ranges of values fairly accurately. For methods and details on noise addition, as well as of insights on the level of protection offered by these methods please see [52].

- Numerical rank swapping [53], [54]: First, values of an attribute value are ranked in ascending order, then each ranked value is swapped with another ranked value randomly chosen within a restricted range (e.g. the rank of two swapped values cannot differ by more than $\mathrm{p} \%$ of the total number of records). This procedure is independently 
used on each variable in the original data set. Thus, numerical rank swapping differs from all other techniques mentioned so far in that it does not perturb the values; it simply swaps the values, breaking associations between a record and its values and also among values in different attributes.

- Shuffling [55]: In data shuffling, the attribute values of an attribute are "permuted" so that, for example, the i-th record in the dataset swaps its value in the attribute with that of the j-th record, the j-th record swaps its value with that of the k-th record etc, in a specific manner that preserves desired data properties, such as relationships between the values that are shuffled. Again, this method does not perturb the values.

The techniques mentioned above primarily focus on microdata protection. For tabular data, (i.e., a statistical table of cells containing count/frequency data, or magnitude data), there is another set of techniques that are reviewed below. In tabular data, count/frequency data represent the number of individuals/respondents who fall into the cell (e.g., the number of individuals with income of a certain level), while magnitude data aggregate values of a particular variable from all individuals in that particular cell (for example the mean income). In the following, we briefly review these methods and refer the reader to [56] for more details about these methods.

- Primary suppression: This operation is applied to sensitive cells (i.e., cells that have been identified as unsafe) and simply removes such cells from the table.

- Secondary suppression: This operation is applied to non-sensitive cells and removes such cells from the table. This is to prevent disclosures that occur due to dependencies between the sensitive sells that are suppressed and other, non-sensitive cells. Such disclosures are possible, for example, when an adversary uses the marginals to estimate the values of the suppressed cells. When secondary suppression needs to delete a subset of cells, among many possible subsets, cells whose removal would not harm data utility substantially are preferred. The harm of a cell to data utility is quantified with a score that is user-specified, and the selection of safe sells to be suppressed is treated as an optimization problem that is solved with an open-source or commercial solver. 
- Table redesign: This operation modifies a table by combining certain rows and columns that are specified by the user. For example, this can be done by adding the cell contents from different columns and rows, thereby aggregating information, in a way that is similar in principle to the recoding operation applied in the context of microdata. While this clearly incurs some data utility loss, the benefit is that it can substantially reduce the number of cells that are suppressed by primary and secondary suppression. Thus, there is a trade-off between the utility loss incurred due to making the variables less specific and the utility gain achieved by reducing the number of cells that must be suppressed.

Implementation of statistical disclosure control techniques in software tools

In this section, we review software for applying statistical disclosure control techniques. Our focus is on software tools that are open-source or closed-source tools, for which there are published details. This is because, for such software tools, there is accessible knowledge about the algorithms implemented in them. Furthermore, we distinguish between techniques applied to microdata and others applied to tabular data. Table 2 below summarizes the techniques applied to microdata and how they are implemented by different software packages.

\begin{tabular}{|l|l|l|}
\hline Technique ISoftware & $\begin{array}{l}\mu \text {-Argus } \\
{[24]}\end{array}$ & $\begin{array}{l}\text { sdcMicro } \\
{[26]}\end{array}$ \\
\hline Recoding & $\mathrm{V}$ & $\mathrm{V}$ \\
\hline Local suppression & Limited & $\mathrm{V}$ \\
\hline PRAM & $\mathrm{V}$ & $\mathrm{V}$ \\
\hline Microaggregation & $\mathrm{V}$ & $\mathrm{V}$ \\
\hline Top/bottom coding & $\mathrm{V}$ & $\mathrm{V}$ \\
\hline Rounding & $\mathrm{V}$ & $\mathrm{V}$ \\
\hline Noise addition & Limited & $\mathrm{V}$ \\
\hline $\begin{array}{l}\text { Numerical } \\
\text { rank swapping }\end{array}$ & Limited & $\mathrm{V}$ \\
\hline Shuffling & & $\mathrm{V}$ \\
\hline
\end{tabular}

Table 2. Statistical Disclosure Control techniques and their implementation in open source software packages. 
As can be seen, each technique is supported by at least one of the two software tools, $\mu$-Argus and sdcMicro. However, sdcMicro additionally supports shuffling and provides more support (e.g., additional methods) for local suppression, noise addition, and numerical rank swapping.

$\mu$-Argus is a software package that has been developed by Statistics Netherlands. Although Statistics Netherlands (Anco Hundepool and Peter-Paul de Wolf) is the main developer, contributions have been made by Josep Domingo-Ferrer (Universitat Rovira i Virgili). $\mu$-ARGUS is currently an Open Source project. The latest version of $\mu$-ARGUS is written in JAVA. The input to the program is microdata along with metadata (e.g. identification level, missing values, length of decimals, attributes to which the same suppression value should be applied). For example, the identification level specifies how identifying is an attribute in a scale from 1 to 3 . The input dataset and the metadata are given as text files. In the $\mu$-Argus tool, the attributes can be numerical or categorical, and there is a Graphical User Interface that helps users with no programming experience work with the tool.

sdcMicro is a package for the statistical software $R$, which has been developed by Statistics Austria and Vienna University of Technology (Matthias Templ, Alexander Kowarik, Bernhard Meindl, Bernd Prantner). The package can be used to quantify the risk of microdata using various estimation methods and to produce protected microdata. The protection is in terms of offering privacy of the underlying respondents. sdcMicro has a Graphical User Interface that is provided through an associated R package called sdcMicroGUI. However, sdcMicro can also be called within the R software, which makes its integration with user-built scripts easy, as long as the user has knowledge of $\mathrm{R}$. Unlike $\mu$-Argus, sdcMicro is suitable for large data sets, as it has more modest requirements in terms of memory and it is also more efficient. The input to sdcMicro is a relational dataset (microdata) that is given as an $\mathrm{R}$ data.frame or as a numeric matrix. Through the functionality of $R$, it is possible to load many different types of files (e.g., CSV, SPSS, etc.) and convert them into matrices or data.frames. Metadata for an input dataset (e.g. names of variables, sampling weights, variables corresponding to sensitive attributes, etc.) are associated to the input, by creating a special class sdcMicroObj in $\mathrm{R}$.

Table 3 below summarizes the techniques applied to tabular data and how they are implemented by different software packages, T-Argus [25] and sdcTable [57]. These are well-known tools for protecting tabular data. We have also included a more specialized tool, G-Confid, which was developed at developed at Statistics Canada [58]. 


\begin{tabular}{|l|l|l|l|}
\hline Technique ISoftware & $\begin{array}{l}\text { T-Argus } \\
{[25]}\end{array}$ & $\begin{array}{l}\text { sdcTable } \\
{[57]}\end{array}$ & $\begin{array}{l}\text { G-Confid } \\
{[58]}\end{array}$ \\
\hline Primary suppression & $\mathrm{V}$ & $\mathrm{V}$ & $\mathrm{V}$ \\
\hline $\begin{array}{l}\text { Secondary } \\
\text { suppression }\end{array}$ & Limited & $\mathrm{V}$ & $\mathrm{V}$ \\
\hline Table redesign & $\mathrm{V}$ & $\mathrm{V}$ & \\
\hline
\end{tabular}

Table 3. Statistical disclosure techniques for tabular data and their implementations in software packages.

As can be seen from the table, both T-Argus and sdcTable support all operations, while G-Confid is a specialized tool that supports only primary and secondary suppression. Before protecting the tabular data, all tools need to identify sensitive cells in a table. A cell of a table is considered sensitive (i.e., unsafe for publication) if it satisfies one of the three following rules: (1) The frequency of a count/frequency cell is below a user-specified threshold. (2) The $n$ largest contributors to a magnitude cell are responsible for $\mathrm{k} \%$ of the total of that cell, where $\mathrm{n}$ and $\mathrm{k}$ are user-specified parameters. (3) The second largest contributor to a magnitude cell can be used to estimate the largest contributor to the cell with precision more than $p \%$, where $p$ is a user-specified parameter. For details on these rules we refer the reader to [59].

We now provide some more information and a brief evaluation of each tool.

T-ARGUS is currently an Open Source project. It is written in Java and can run in the Windows environment. It supports both free and commercial Linear Programming (LP) solvers, such as CPLEX, for secondary cell suppression. It also offers a Graphical User Interface that controls the flow of the program (e.g., identification of sensitive cells, protection through various techniques, and assessment of the results) and makes the software easy to use. Useful features of the tool are its ability to get microdata as input and perform tabulation (i.e., convert the data into tabular format) automatically while protecting the data, and to preform recoding on the fly. It is also customizable, in terms of defining data utility, as the cost of user-specified costs for the suppression of individual cells.

sdcTable is currently also an Open Source project. It is written as a package for the statistical software $\mathrm{R}$. This makes it relatively easy, for users with experience in $\mathrm{R}$, to use sdcTable within 
their own programs. It also has a graphical user interface for users with no experience in R. One advantage of sdcTable is that it can employ the free LP-solver that is part of R for secondary cell suppression.

G-Confid is a tool developed at Statistics Canada. Different from t-Argus and sdcTable, it is not a stand-alone tool but it requires the commercial SAS software suite (it requires SAS version 9.2). G-Confid can be used to construct or evaluate cell suppression patterns for tabular data, and it has support for secondary suppression through the SAS/OR Linear Programming (LP) solver. A benefit of G-Confid is that it exploits SAS to scale to large tables.

\section{Pseudonymisation}

Distinct from anonymization, which involves permanently removing any identifiable information from a record, pseudonymisation involves the replacement of identifiable fields (direct identifiers) in a dataset with a pseudonym. It is described in the GDPR legislation [60] as "the processing of personal data in such a manner that the personal data can no longer be attributed to a specific data subject without the use of additional information." Those additional information should be kept separately. Pseudonymidsation is applied to microdata.

One reason for undertaking pseudonymisation is that datasets held on servers often need to be linked to provide utility, and this linkage requires some identifiable (i.e. disclosive) attributes within each dataset to ensure the linking is done correctly. One way of doing this is by removing identifying attributes (for example name, address or location) through anonymization but this poses problems for the linkage of data held in a distributed environment. The ICO code of practice [61] recognizes the value of pseudonymisation for linkage in that "pseudonymised or de-identified data may be very valuable to researchers because of its individual-level granularity and because pseudonymised records from different sources can be relatively easy to match." We will focus on the widely used pseudonymisation technique of hashing.

\section{Hashing}

Hashing involves replacing identifiable information with a pseudonymised string called the digest. The Secure Hash Algorithm version 2 (SHA-2) is the current standard, created by the US National Security Agency (NSA) and published by the National Institute of Standards and Technology. 
SHA-2 consists of a range of hash functions (of different bit-lengths) which are deterministic procedures that takes input, for example names, addresses or date of birth and returns an output that was intentionally changed by an algorithm to something which is not personally identifiable. Due to its deterministic attributes and the inability to reverse-engineer a hashed value, it has been widely adopted for security applications and procedures. For example 256 bit SHA is standard in (Secure Sockets Layer) SSL encryption for documents sent over the internet.

The deterministic nature of hashing means that, given the same original input value and hashing function, they will always provide the same output. This is important for linking datasets, since the same digest can be generated for multiple datasets where the original input values held within a secure research environment. In terms of data dissemination, including the hashed value as part of the release table allows users to request further information without jeopardizing the security of the dataset. It also allows for the follow-up of an individual record within a safe environment. It can be combined with anonymization techniques to provide different levels of security for datasets.

Being deterministic does also mean however that hashing is vulnerable to dictionary or bruteforce attack, whereby hashes are generated of many possible inputs and then matched to the released digest in order to attempt to reverse engineer the content. One way to add an additional layer of protection is to introduce 'salt' to the hash. This involves adding extra data (salt) to the input column to be pseudonymised which means that someone with the same original information could not create the same digest. Crucially, for the digest with salt to be reproduced within a different secure research environment (in order to enable linkage) the salt file needs to be encrypted and shared. The salt file should be stored securely for it to offer any additional protection. Adding salt to the digest will not fully protect against a brute force/ dictionary attack but will make it very computationally expensive. The longer the salt the more expensive this becomes.

A hash can be combined with a cryptographic key to produce a Hash-based Message Authentication Code (HMAC). The key provides an additional level of protection since, when properly implemented, reconstructing the digest is possible only with access to the key. Security is only provided if the key is held securely within a research environment. This is an approach which has been applied by ONS during the Beyond 2011 Programme of methodological development [62]. The ONS implementation has drawn criticism [63], partly because the 
construction of a different key for each field (first name, last name, date of birth, postcode) left the approach open to frequency attack - the ubiquity of the surname Smith is used to exemplify this as analyzing the frequency of HMAC tabs would identify this. Criticism was also levelled at the publication of 'similarity tables' [64] which revealed the uniqueness of match keys across each of the fields. It was argued [63] that using public information such a s a list of names, similarity scores could be recalculated and as such the tables should not be published outside of a secure environment. More broadly, this demonstrates that information in the public domain can be leveraged to exploit weaknesses in hashing methodologies, and care should be taken to ensure that they are properly implemented, in conjunction with secure research infrastructure.

Since the introduction of GDPR and recommendations around the pseudonymisation of data [60] there appear to be a range of software solutions proclaiming to help organisations be compliant. Many of these are proprietary software. There are also a number of open source tools including: HashTool [65], a Windows based freeware utility with drag-and-drop functionality which creates hash values using a choice of hashing algorithms from SHA2 and Quick Hash GUI [66] with similar functionality for multiple platforms. More documentation is available with Open Pseudonymiser, an open source software with an accompanying desktop application for undertaking hashing, developed by researchers at the University of Nottingham [67]. Open Pseudonymiser provides the tools to produce a digest using a SHA2 256-bit hashing function as well as a random 128 character salt file. It is issued under the GNU General Public License. The pseudonymisation at source nature of Open Pseudonymiser means that the data controller retains control of the process. In brief, Open Pseudonymiser:

- Creates a digest of the required field of a csv using a one-way hashing algorithm

- Allows the user to create encrypted salt file which can be added to the input column

- The salt files can be served via a Key Server to other users

- Provides a tool for validating NHS numbers if these are intended to be used in the creation of the digest

\section{Conclusions and directions for future work}

This chapter provided an overview of methods for anonymization of microdata, set-valued data, and both microdata and set-valued data, covering mainly algorithms that have been implemented as open source software packages. For microdata, we have provided a classification of algorithms, based on the way they explore the space of possible anonymization solutions and 
provided examples of algorithms of each category - specifically, the following six popular algorithms: Incognito, Top-down Specialization, Mondrian, Cluster, and OLA. We then briefly discussed implementations of these algorithms in six software tools, namely SECRETA, UTDA, TIAMAT, CAT, and ARX. In conclusion, SECRETA is the tool that implements most algorithms, with the exception of OLA, which is a specialized variant of Incognito designed for biomedical data, while ARX is the most flexible tool in terms of providing access to a large set of models of measuring disclosure risk. For set-valued data, we have provided a classification of algorithms, based on the way they work and then provided six examples of popular algorithms: Apriori Anonymization, LRA, VPA, COAT, and PCTA. For datasets containing a combination of microdata (relational attributes) and set-valued attributes, we provided three frameworks that can be instantiated with different algorithms for anonymizing microdata and set-valued data.

From our review of the methods and software, it can be seen that algorithms that offer protection from identity and/or attribute disclosure are hardly implemented by any tool, and the support for algorithms that anonymize set-valued data and data containing both relational and set-valued attributes is currently only provided by the SECRETA tool. In addition, no tool offers support for anonymizing large-scale data (i.e., data that does not fit into the memory of a single computer) through the use of parallel anonymization algorithms. These two limitations suggest interesting directions for future work. In addition, there is a multitude of other types of data, including data streams and graphs, whose anonymization is interesting and currently not feasible by opensource (or closed-source, to the best of our knowledge) tools. For these, more complex, types of data, it would also be interesting to see anonymization software.

The chapter also provided an overview of statistical disclosure control methods, covering both methods for microdata, as well as methods for tabular data, with an emphasis on methods that have been implemented in open-source software packages. For microdata protection, there are data transformation operations such as recording, local suppression, PRAM, microaggregation, top/bottom coding, rounding, noise addition, numerical rank swapping and shuffling. These operations are supported by open-source software tools, $\mu$-Argus and sdcMicro. Each of these tools offers a graphical user interface and various methods to perform the operations. For tabular data protection, we have discussed operations for identifying cells that need protection and operations for protecting the data, such as primary suppression, secondary suppression, and 
table redesign, as they are implemented by open-source software such as T-Argus, sdcTable, and G-confid.

We provided a brief overview of Pseudonymisation, which is distinct from anonymization in that it is a process for retaining individual level data but allows for the recovery of personal information. Pseudonymisation is particularly useful if there is a need to link datasets across different servers or locations. Of the algorithms used for Pseudonymisation, Hashing is by far the most widely implemented, and has standards which should be adhered to. Key to ensuring data integrity and security when using Pseudonymisation is appropriate secure infrastructure where original data, salt files and, in the case of HMAC, decryption keys can be stored. We have highlighted recent criticism of hashing techniques which arise from a miss-understanding of the security that the approach offers, demonstrating a need to fully understand a technology solution before it is implemented.

\section{References}

[1] "https://www.ons.gov.uk/surveys." .

[2] J. Han, M. Kamber, and J. P. Professor, Data Mining: Concepts and Techniques, 3 edition. Haryana, India; Burlington, MA: Morgan Kaufmann, 2011.

[4] Opus International Consultants, "Public attitudes to data integration: Report prepared for Statistics New Zealand," 2015. [Online]. Available: http://archive.stats.govt.nz/browse_for_stats/snapshots-of-nz/integrated-datainfrastructure/keep-data-safe/public-attitudes-data-integration-2015.aspx.

[5] A. Gkoulalas-Divanis, G. Loukides, L. Xiong, and J. Sun, "Informatics methods in medical privacy," J. Biomed. Inform., vol. 50, pp. 1-3, 2014.

[6] C. Aggarwal and P. S. Yu, Privacy-Preserving Data Mining - Models and Algorithms. Springer US.

[7] K. H. Jones et al., "A case study of the Secure Anonymous Information Linkage (SAIL) Gateway: A privacy-protecting remote access system for health-related research and evaluation," J. Biomed. Inform., vol. 50, pp. 196-204, Aug. 2014.

[8] B. C. M. Fung, K. Wang, R. Chen, and P. S. Yu, "Privacy-preserving Data Publishing: A Survey of Recent Developments," ACM Comput Surv, vol. 42, no. 4, pp. 14:1-14:53, Jun. 2010.

[9] G. Poulis, S. Skiadopoulos, G. Loukides, and A. Gkoulalas, "Apriori-based algorithms for km-anonymizing trajectory data," Trans. Data Privacy, vol. 7, no. 2, pp. 165-194, 2014.

[10] R. Shokri, G. Theodorakopoulos, and C. Troncoso, "Privacy games along location traces: A game-theoretic framework for optimizing location privacy," ACM Trans. Priv. Secur., vol. 19, pp. 11:1-11:31, Dec. 2016.

[11] A. Narayanan and V. Shmatikov, "De-anonymizing Social Networks," in 2009 30th IEEE Symposium on Security and Privacy, Oakland, CA, USA, 2009, pp. 173-187.

[12] K. LeFevre, D. J. DeWitt, and R. Ramakrishnan, "Incognito: Efficient Full-domain Kanonymity," in Proceedings of the 2005 ACM SIGMOD International Conference on Management of Data, New York, NY, USA, 2005, pp. 49-60. 
[13] M. Terrovitis, N. Mamoulis, and P. Kalnis, "Privacy-preserving anonymization of setvalued data," Proc. VLDB Endow., vol. 1, no. 1, pp. 115-125, Aug. 2008.

[14] M. Terrovitis, N. Mamoulis, and P. Kalnis, "Local and global recoding methods for anonymizing set-valued data," VLDB J., vol. 20, no. 1, pp. 83-106, Feb. 2011.

[15] J. Cao, P. Karras, C. Raïssi, and K.-L. Tan, " $\rho$-uncertainty: inference-proof transaction anonymization," Proc. VLDB Endow., vol. 3, no. 1-2, pp. 1033-1044, Sep. 2010.

[16] G. Loukides, A. Gkoulalas-Divanis, and B. Malin, "COAT: COnstraint-based anonymization of transactions," Knowl. Inf. Syst., vol. 28, no. 2, pp. 251-282, Aug. 2011.

[17] G. Poulis, G. Loukides, S. Skiadopoulos, and A. Gkoulalas-Divanis, "Anonymizing datasets with demographics and diagnosis codes in the presence of utility constraints," $J$. Biomed. Inform., vol. 65, pp. 76-96, Jan. 2017.

[18] G. Poulis, G. Loukides, A. Gkoulalas-Divanis, and S. Skiadopoulos, "Anonymizing Data with Relational and Transaction Attributes," in Machine Learning and Knowledge Discovery in Databases, 2013, pp. 353-369.

[19] G. Poulis, A. Gkoulalas-Divanis, G. Loukides, S. Skiadopoulos, and C. Tryfonopoulos, "SECRETA: A System for Evaluating and Comparing RElational and Transaction Anonymization algorithms." OpenProceedings.org, 2014.

[20] "UTD Anonymization ToolBox." [Online]. Available: http://www.cs.utdallas.edu/dspl/cgi-bin/toolbox/index.php. [Accessed: 25-Oct-2018].

[21] X. Xiao, G. Wang, and J. Gehrke, "Interactive anonymization of sensitive data," in Proceedings of the 35th SIGMOD international conference on Management of dataSIGMOD '09, Providence, Rhode Island, USA, 2009, p. 1051.

[22] "ARX - Data Anonymization Tool | A comprehensive software for privacy-preserving microdata publishing." [Online]. Available: https://arx.deidentifier.org/. [Accessed: 25-Oct2018].

[23] "Statistical Disclosure Control," Wiley.com. [Online]. Available: https://www.wiley.com/en-gb/Statistical+Disclosure+Control-p-9781118348215. [Accessed: 25-Oct-2018].

[24] “ $\mu$-ARGUS.” [Online]. Available: http://research.cbs.nl/casc/mu.htm. [Accessed: 25-Oct$2018]$.

[25] “ $\tau$-ARGUS.” [Online]. Available: http://research.cbs.nl/casc/tau.htm. [Accessed: 25-Oct2018].

[26] "Statistical Disclosure Control (sdcMicro) | IHSN." [Online]. Available: http://www.ihsn.org/software/disclosure-control-toolbox. [Accessed: 25-Oct-2018].

[27] A. Machanavajjhala, J. Gehrke, D. Kifer, and M. Venkitasubramaniam, "L-diversity: privacy beyond k-anonymity," in 22nd International Conference on Data Engineering (ICDE'06), 2006, pp. 24-24.

[28] L. Sweeney, "K-anonymity: A Model for Protecting Privacy," Int J Uncertain Fuzziness Knowl-Based Syst, vol. 10, no. 5, pp. 557-570, Oct. 2002.

[29] A. Gkoulalas-Divanis, G. Loukides, and J. Sun, "Publishing data from electronic health records while preserving privacy: A survey of algorithms," J. Biomed. Inform., vol. 50, pp. 4-19, Aug. 2014.

[30] C. Dwork and A. Roth, "The Algorithmic Foundations of Differential Privacy," Found. Trends Theor. Comput. Sci., vol. 9, no. 3-4, pp. 211-407, 2013.

[31] J. Liu, L. Xiong, and J. Luo, "Semantic Security: Privacy Definitions Revisited," Trans. Data Privacy, vol. 6, pp. 185-198, 2013. 
[32] J. Lee and C. Clifton, "Differential Identifiability," in Proceedings of the 18th ACM SIGKDD International Conference on Knowledge Discovery and Data Mining, New York, NY, USA, 2012, pp. 1041-1049.

[33] L. Sweeney, "k-ANONYMITY: A MODEL FOR PROTECTING PRIVACY," Int. $J$. Uncertain. Fuzziness Knowl.-Based Syst., vol. 10, no. 05, pp. 557-570, Oct. 2002.

[34] R. C.-W. Wong, J. Li, A. W.-C. Fu, and K. Wang, "(\$lalpha\$, K)-anonymity: An Enhanced K-anonymity Model for Privacy Preserving Data Publishing," in Proceedings of the 12th ACM SIGKDD International Conference on Knowledge Discovery and Data Mining, New York, NY, USA, 2006, pp. 754-759.

[35] T. M. Truta and B. Vinay, "Privacy Protection: P-Sensitive k-Anonymity Property," in Proceedings of the 22Nd International Conference on Data Engineering Workshops, Washington, DC, USA, 2006, pp. 94-.

[36] N. Li, T. Li, and S. Venkitasubramaniam, "t-Closeness: Privacy Beyond k-Anonymity and 1-Diversity," in Proceedings of the 22Nd International Conference on Data Engineering, pp. 106-115.

[37] G. Loukides, A. Gkoulalas-Divanis, and J. Shao, "Efficient and flexible anonymization of transaction data," Knowl. Inf. Syst., vol. 36, no. 153, pp. 153-210.

[38] J. Domingo-Ferrer, A. Martínez-Ballesté, J. M. Mateo-Sanz, and F. Sebé, "Efficient Multivariate Data-oriented Microaggregation," VLDB J., vol. 15, no. 4, pp. 355-369, Nov. 2006.

[39] G. Loukides, "Data utility and privacy protection in data publishing," $\mathrm{PhD}$ thesis, Cardiff University, 2008.

[40] T. Li and N. Li, "Optimal k-Anonymity with Flexible Generalization Schemes through Bottom-up Searching," in Sixth IEEE International Conference on Data Mining - Workshops (ICDMW'06), Hong Kong, China, 2006, pp. 518-523.

[41] B. C. M. Fung, Ke Wang, and P. S. Yu, "Top-Down Specialization for Information and Privacy Preservation," in 21st International Conference on Data Engineering (ICDE'05), Tokyo, Japan, 2005, pp. 205-216.

[42] B. C. M. Fung, K. Wang, and P. S. Yu, "Anonymizing Classification Data for Privacy Preservation,” IEEE Trans. Knowl. Data Eng., vol. 19, no. 5, pp. 711-725, May 2007.

[43] X. Zhang, C. Liu, S. Nepal, C. Yang, W. Dou, and J. Chen, "Combining Top-Down and Bottom-Up: Scalable Sub-tree Anonymization over Big Data Using MapReduce on Cloud," in 2013 12th IEEE International Conference on Trust, Security and Privacy in Computing and Communications, 2013, pp. 501-508.

[44] X. Zhang, C. Liu, S. Nepal, C. Yang, W. Dou, and J. Chen, "A hybrid approach for scalable sub-tree anonymization over big data using MapReduce on cloud," J. Comput. Syst. Sci., vol. 80, no. 5, pp. 1008-1020, Aug. 2014.

[45] K. LeFevre, D. J. DeWitt, and R. Ramakrishnan, "Mondrian Multidimensional KAnonymity," in Proceedings of the 22Nd International Conference on Data Engineering, Washington, DC, USA, 2006, pp. 25-.

[46] J.-W. Byun, A. Kamra, E. Bertino, and N. Li, "Efficient k-Anonymization Using Clustering Techniques," in Advances in Databases: Concepts, Systems and Applications, 2007, pp. 188-200.

[47] A. Gkoulalas-Divanis and G. Loukides, "PCTA: privacy-constrained clustering-based transaction data anonymization," in Proceedings of the 4th International Workshop on 
Privacy and Anonymity in the Information Society - PAIS '11, Uppsala, Sweden, 2011, pp. $1-10$.

[48] C. Dai, G. Ghinita, E. Bertino, J.-W. Byun, and N. Li, "TIAMAT: A Tool for Interactive Analysis of Microdata Anonymization Techniques," Proc VLDB Endow, vol. 2, no. 2, pp. 1618-1621, Aug. 2009.

[49] A. G. de Waal, "Optimal Local Suppression in Microdata," Journal of Official Statistics, vol. 14, no. 4, pp. 421-435, 1998.

[50] J. M. Gouweleeuw and P. Kooiman, "Post Randomisation for Statistical Disclosure Control: Theory and Implementation," p. 16.

[51] J. Domingo-Ferrer and J. M. Mateo-Sanz, "Practical data-oriented microaggregation for statistical disclosure control - IEEE Journals \& Magazine," IEEE Trans on Knowl. and Data Engin., vol. 14, pp. 189-201, 2002.

[52] J. Domingo-Ferrer, F. Sebé, and J. Castellà-Roca, "On the Security of Noise Addition for Privacy in Statistical Databases," in Privacy in Statistical Databases, 2004, pp. 149-161.

[53] U. C. Bureau, "Controlled Data-Swapping Techniques for Masking Use Microdata Sets." [Online]. Available: https://www.census.gov/library/working-papers/1996/adrm/rr96-

04.html. [Accessed: 26-Oct-2018].

[54] S. E. Fienberg and J. McIntyre, "Data Swapping: Variations on a Theme by Dalenius and Reiss," in Privacy in Statistical Databases, vol. 3050, J. Domingo-Ferrer and V. Torra, Eds. Berlin, Heidelberg: Springer Berlin Heidelberg, 2004, pp. 14-29.

[55] K. Muralidhar and R. Sarathy, "Data Shuffling: A New Masking Approach for Numerical Data," Manag. Sci., vol. 52, no. 5, pp. 658-670, 2006.

[56] L. Willenborg and T. de Waal, "Application of Non-Perturbative Techniques for Tabular Data," in Elements of Statistical Disclosure Control, L. Willenborg and T. de Waal, Eds.

New York, NY: Springer New York, 2001, pp. 175-217.

[57] B. Meindl and M. Temple, "A computational framework to protect tabular data," in Joint UNECE/Eurostat work session on statistical data confidentiality, 2011.

[58] P. J.-L. Tambay and J.-M. Fillion, "New business survey confidentiality software GConfid," in UNECE work session on statistical data confidentiality, 2011, p. 11.

[59] A. Sukasih, D. Jang, and D. Edson, "Using Tau-Argus and sdcTable to Conduct Secondary Cell Supression for Linked Tables," Mathematica Policy Research, Mathematica Policy Research Reports, Aug. 2011.

[60] “Article 4 EU GDPR 'Definitions,"” 2016.

[61] ICO, “Anonymisation: managing data protection risk code of practice,” p. 108.

[62] Office for National Statistics, "Beyond 2011 safeguarding data for research: Our policy M10," 2013.

[63] C. Culnane, B. I. P. Rubinstein, and V. Teague, "Vulnerabilities in the use of similarity tables in combination with pseudonymisation to preserve data privacy in the UK Office for National Statistics' Privacy-Preserving Record Linkage," ArXiv171200871 Cs, Dec. 2017.

[64] Office for National Statistics, "Beyond 2011 matching anonymous data M9," 2013.

[65] digitalvolcano software, "Hash Tool - Calculate File Hashes." [Online]. Available: https://www.digitalvolcano.co.uk/hash.html. [Accessed: 26-Oct-2018].

[66] "Quickhash GUI - Cross-platform data hashing tool for Windows, Linux and OSX." [Online]. Available: https://quickhash-gui.org/. [Accessed: 26-Oct-2018]. 
[67] "OpenPseudononymiser." [Online]. Available:

https://www.openpseudonymiser.org/OpenPseudonymiser_Docs.aspx. [Accessed: 26-Oct2018].

[68] El Emam K, Dankar FK, Issa R, Jonker E, Amyot D, Cogo E, Corriveau JP, Walker M, Chowdhury S, Vaillancourt R, Roffey T. A globally optimal k-anonymity method for the deidentification of health data. Journal of the American Medical Informatics Association. 2009 Sep 1;16(5):670-82.

[69] Prasser F, Kohlmayer F. Putting statistical disclosure control into practice: The ARX data anonymization tool. In Medical Data Privacy Handbook 2015 (pp. 111-148). Springer, Cham. 